University of Wollongong

Research Online

Australian Institute for Innovative Materials -

Papers

Australian Institute for Innovative Materials

$1-1-2019$

Simultaneous tuning of magnetocrystalline anisotropy and spin

reorientation transition via Cu substitution in $\mathrm{Mn}-\mathrm{Ni}-\mathrm{Ga}$ magnets for

nanoscale biskyrmion formation

Guizhou Xu

Nanjing University of Science And Technology

Yurong You

Nanjing University of Science And Technology

Jiaxuan Tang

Nanjing University of Science And Technology

Hongguo Zhang

Beijing University of Technology

Hang Li

Chinese Academy Of Sciences

See next page for additional authors

Follow this and additional works at: https://ro.uow.edu.au/aiimpapers

Part of the Engineering Commons, and the Physical Sciences and Mathematics Commons

Research Online is the open access institutional repository for the University of Wollongong. For further information contact the UOW Library: research-pubs@uow.edu.au 


\title{
Simultaneous tuning of magnetocrystalline anisotropy and spin reorientation transition via Cu substitution in Mn-Ni-Ga magnets for nanoscale biskyrmion formation
}

\author{
Abstract \\ Skyrmions with multiple helicity or topology in centrosymmetric crystals are intriguing magnetic-domain \\ objects due to their diverse dynamics under external stimuli. Here we illustrate how the two key gradients \\ of magnetocrystalline anisotropy (MCA) and spin reorientation transition (SRT) affect the skyrmion \\ formation and topology by Cu substitution in the biskyrmion-host MnNiGa alloy. The MCA and SRT are \\ simultaneously tuned in a large scope, while the original high Curie temperature (TC) is retained. Detailed \\ neutron-scattering studies revealed the construction of a noncollinear canted magnetic structure below \\ the SRT temperature (TSR), which effectively correlates the SRT with the evolution of the MCA, as well as \\ the exchange interaction. The Cu substitution raises the TSR to merge with the TC, and meanwhile, \\ reduces the c-axis anisotropy. Lorentz transmission electron microscopy revealed the formation of \\ stacked biskyrmions from above room temperature to lower temperatures in $\mathrm{MnNi1}-\mathrm{xCuxGa}(\mathrm{x}=0-0.3)$ in \\ the presence of proper MCA. Micromagnetic simulations further confirmed the great effect of uniaxial \\ anisotropy on the stabilization of biskyrmions. Our work has helped clarify the evolution of magnetic \\ structures and their correlation to the SRT, providing an account of the effect of MCA and exchange \\ interaction on the biskyrmion formation. \\ Disciplines \\ Engineering | Physical Sciences and Mathematics \\ Publication Details \\ Xu, G., You, Y., Tang, J., Zhang, H., Li, H., Miao, X., Gong, Y., Hou, Z., Cheng, Z., Wang, J., Studer, A. J., Xu, F. \\ \& Wang, W. (2019). Simultaneous tuning of magnetocrystalline anisotropy and spin reorientation \\ transition via Cu substitution in Mn-Ni-Ga magnets for nanoscale biskyrmion formation. Physical Review \\ B: Covering condensed matter and materials physics, 100 (5), 054416-1-054416-8.
}

\section{Authors}

Guizhou Xu, Yurong You, Jiaxuan Tang, Hongguo Zhang, Hang Li, Xuefei Miao, Yuanyuan Gong, Zhipeng Hou, Zhenxiang Cheng, Jian Li Wang, Andrew J. Studer, Feng Xu, and Wenhong Wang 


\title{
Simultaneous tuning of magnetocrystalline anisotropy and spin reorientation transition via $\mathrm{Cu}$ substitution in Mn-Ni-Ga magnets for nanoscale biskyrmion formation
}

\author{
Guizhou Xu $\odot,{ }^{1}$ Yurong You, ${ }^{1}$ Jiaxuan Tang, ${ }^{1}$ Hongguo Zhang, ${ }^{2}$ Hang Li,${ }^{5}$ Xuefei Miao, ${ }^{1}$ Yuanyuan Gong, ${ }^{1}$ \\ Zhipeng Hou, ${ }^{5}$ Zhenxiang Cheng, ${ }^{3,}{ }^{*}$ Jianli Wang, ${ }^{4}$ Andrew J. Studer, ${ }^{4}$ Feng Xu, ${ }^{1, \dagger}$ and Wenhong Wang ${ }^{5, *}$ \\ ${ }^{1}$ MIIT Key Laboratory of Advanced Metallic and Intermetallic Materials Technology, School of Materials Science and Engineering, \\ Nanjing University of Science and Technology, Nanjing 210094, China \\ ${ }^{2}$ College of Materials Science and Engineering, Beijing University of Technology, Beijing 100124, China \\ ${ }^{3}$ Institute for Superconducting and Electronic Materials, Innovation Campus, University of Wollongong, \\ Squires Way, North Wollongong, New South Wales 2500, Australia \\ ${ }^{4}$ Australian Nuclear Science and Technology Organization (ANSTO), Lucas Heights, New South Wales 2234, Australia \\ ${ }^{5}$ Beijing National Laboratory for Condensed Matter Physics, Institute of Physics, Chinese Academy of Sciences, Beijing 100190, China
}

(Received 22 May 2019; revised manuscript received 31 July 2019; published 13 August 2019)

\begin{abstract}
Skyrmions with multiple helicity or topology in centrosymmetric crystals are intriguing magnetic-domain objects due to their diverse dynamics under external stimuli. Here we illustrate how the two key gradients of magnetocrystalline anisotropy (MCA) and spin reorientation transition (SRT) affect the skyrmion formation and topology by $\mathrm{Cu}$ substitution in the biskyrmion-host MnNiGa alloy. The MCA and SRT are simultaneously tuned in a large scope, while the original high Curie temperature $\left(T_{C}\right)$ is retained. Detailed neutron-scattering studies revealed the construction of a noncollinear canted magnetic structure below the SRT temperature $\left(T_{\mathrm{SR}}\right)$, which effectively correlates the SRT with the evolution of the MCA, as well as the exchange interaction. The $\mathrm{Cu}$ substitution raises the $T_{\mathrm{SR}}$ to merge with the $T_{\mathrm{C}}$, and meanwhile, reduces the $c$-axis anisotropy. Lorentz transmission electron microscopy revealed the formation of stacked biskyrmions from above room temperature to lower temperatures in $\mathrm{MnNi}_{1-x} \mathrm{Cu}_{x} \mathrm{Ga}(x=0-0.3)$ in the presence of proper MCA. Micromagnetic simulations further confirmed the great effect of uniaxial anisotropy on the stabilization of biskyrmions. Our work has helped clarify the evolution of magnetic structures and their correlation to the SRT, providing an account of the effect of MCA and exchange interaction on the biskyrmion formation.
\end{abstract}

DOI: 10.1103/PhysRevB.100.054416

\section{INTRODUCTION}

Magnetic skyrmions are nanoscale topological particles with a whirling spin structure, holding great potential as information carriers for the realization of high-density and low-power-consumption spintronic devices [1-3]. Besides in the typical noncentrosymmetric materials [4-6] or interface of magnetic multilayers [7-9] with Dzyaloshinskii-Moriya interaction, skyrmions in the centrosymmetric systems are attracting growing attention due to their relatively high Curie temperature and wide temperature stability [10-14]. In addition, they can exhibit additional degrees of freedom of helicity and vorticity (or topology), offering the opportunity to study their intriguing diverse dynamics under external stimuli $[15,16]$.

Among them, the biskyrmion state, with topological charge of 2 , has been predicted to reveal a unique helicity lock-unlock transition under external current [16]. It has been identified in several materials, including the thin plates of the layered magnetite $\mathrm{La}_{2-2 x} \mathrm{Sr}_{1+2 x} \mathrm{Mn}_{2} \mathrm{O}_{7}$ [11], the $\mathrm{Ni}_{2}$ In-type $\mathrm{MnNiGa}$ alloy

\footnotetext{
*Corresponding author: cheng@uow.edu.au

${ }^{\dagger}$ xufeng@njust.edu.cn

†wenhong.wang@iphy.ac.cn
}

[12,17-20], single crystals of $\mathrm{Cr}_{11} \mathrm{Ge}_{19}$ [21], and very recently in MnPdGa [22]. The three-dimensional spin distribution and anisotropy-dependent orientation of biskyrmions have also been uncovered by recent small-angle neutron diffraction measurements on $\mathrm{MnNiGa}$ [23]. However, a general formation mechanism of biskyrmions is still ambiguous. Yao et al. [24] and Loudon et al. [25] have argued that the biskyrmions under Lorentz transmission electron microscopy (TEM) can possibly be depicted as an inclined type-II (topological trivial) bubble. But we notice that there are distinguished features in the Lorentz TEM images of biskyrmion and its trivial bubble that are obtained in the same material, for example, in the different crystal orientations of $\left(\mathrm{Mn}_{1-x} \mathrm{Ni}_{x}\right)_{65} \mathrm{Ga}_{35}$ [26] or in the small-Ga-substituted MnNiGa alloys [20]. Therefore, as an important type of skyrmion state, its formation origin and stability condition deserves in-depth study.

It is known that magnetocrystalline anisotropy (MCA) plays important role in producing skyrmions in the centrosymmetric materials. But for the biskyrmion, the condition can be more strict considering the narrow family of it. Besides the MCA, we noticed that a spin reorientation transition (SRT) is present in most of the existent materials that host skyrmions, such as the $M$-type barium ferrite [10,27], the layered $\mathrm{La}_{2-2 x} \mathrm{Sr}_{1+2 x} \mathrm{Mn}_{2} \mathrm{O}_{7}$ [11,28], Mn-Pt-Sn [29], and hexagonal MnNiGa [12]. It was confirmed that the 

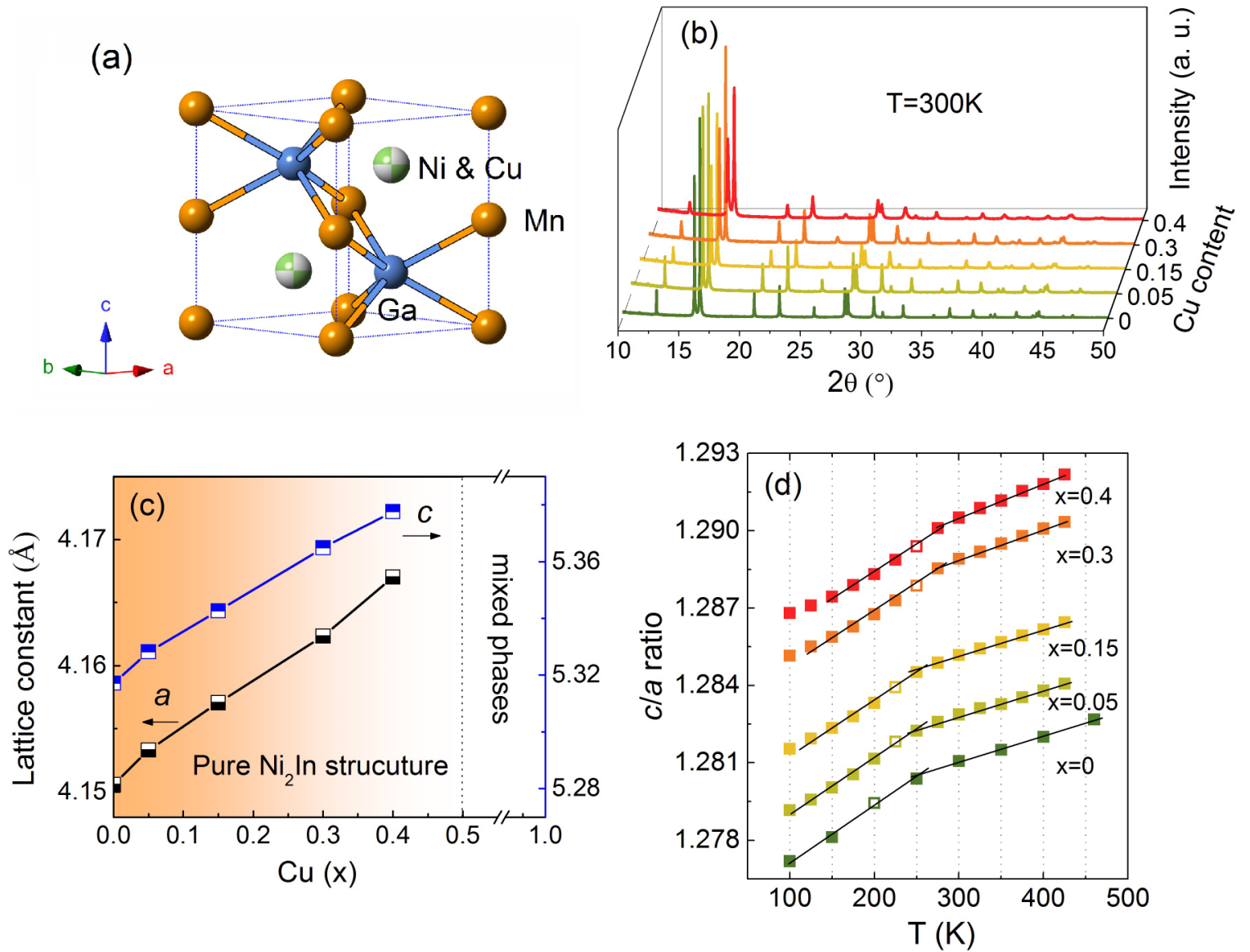

FIG. 1. (a) Hexagonal crystal structure of $\mathrm{MnNi}_{1-x} \mathrm{Cu}_{x} \mathrm{Ga}$, where $\mathrm{Cu}$ occupies the $\mathrm{Ni}$ site. The exact atomic position is determined by neutron diffraction. (b) RT synchrotron XRD patterns for $x=0,0.05,0.15,0.3,0.4$. (c) Variation of the lattice constant with Cu content and indication of the single-phase region. (d) Temperature dependence of the $c / a$ ratios for various compositions.

SRT of the layered manganite $\mathrm{La}_{2-2 x} \mathrm{Sr}_{1+2 x} \mathrm{Mn}_{2} \mathrm{O}_{7}[11,28]$ affects the magnetic-domain structure. Thus understanding the underneath magnetic structures and their mutual correlation between the MCA and SRT becomes fundamentally important to the understanding of the biskyrmion formation.

In this work, by a continuous tuning of the magnetic structure with $\mathrm{Cu}$ substitution of $\mathrm{Ni}$ atom in MnNiGa, we established a rather definite relation between the MCA and SRT. Detailed neutron diffractions were performed to study the spin configuration in an atomic scale, and Lorentz TEM were applied to investigate the real-space magnetic domains in a mesoscopic scale, respectively. A noncollinear canted ferromagnetic structure is constructed at temperatures below the SRT temperature, which continually grows upon $\mathrm{Cu}$ substitution until it nearly merges with the Curie temperature (at $x=0.4$ in $\mathrm{MnNi}_{1-x} \mathrm{Cu}_{x} \mathrm{Ga}$ ). Enhancement of the SRT temperature is found to be intimately associated with the reduction of the anisotropy. Correspondingly, the biskyrmion states are preserved for $x=0-0.3$ and vanished at $x=0.4$, caused by the significant lowering of the MCA. In a combination of micromagnetic simulations, we have revealed the effect of both the magnitude and direction of the MCA on the stability of a biskyrmion. On a more general note, our study provides an important benchmark of the effects of both the underlying magnetic structure and the macroscopy magnetic parameters on biskyrmion formation in materials featuring SRT.

\section{METHODS}

A series of bulk polycrystalline ingots with nominal compositions of $\mathrm{MnNi}_{1-x} \mathrm{Cu}_{x} \mathrm{Ga} \quad(x=0-1.0)$ were synthesized by arc melting mixtures of highly pure $\mathrm{Mn}, \mathrm{Ni}$, and Ga metals in a pure argon atmosphere. Phase purity and crystal structure of the alloys were examined by using both the $\mathrm{Cu} \mathrm{Ka}(\lambda=1.54 \AA)$ x-ray powder diffraction and high-resolution synchrotron x-ray powder diffraction at the Australian Synchrotron facility $(\lambda=0.59 \AA)$.

Neutron powder diffraction at various temperatures was performed on the Wombat (high-intensity powder diffractometer) at the OPAL facility (Lucas Height, Australia), applying a constant neutron wavelength of $1.622 \AA$ A. Refinements of the x-ray diffraction and neutron patterns were performed using the Rietveld method, and the irreducible representation analysis of the magnetic structure was carried out using the BASIREPS program, both implemented in the FULLPROF package $[30,31]$.

The magnetization properties were measured with the vibrating sample magnetometer (VSM) accessory of the physical property measurement system (PPMS, Quantum Design). In order to examine the MCA of the polycrystalline samples, the method of magnetic field alignment was applied. The bulk ingots were ground to powders and mixed with the glue (3M Scotch-Weld, Epoxy Adhesive DP 105 Clear), then solidified 

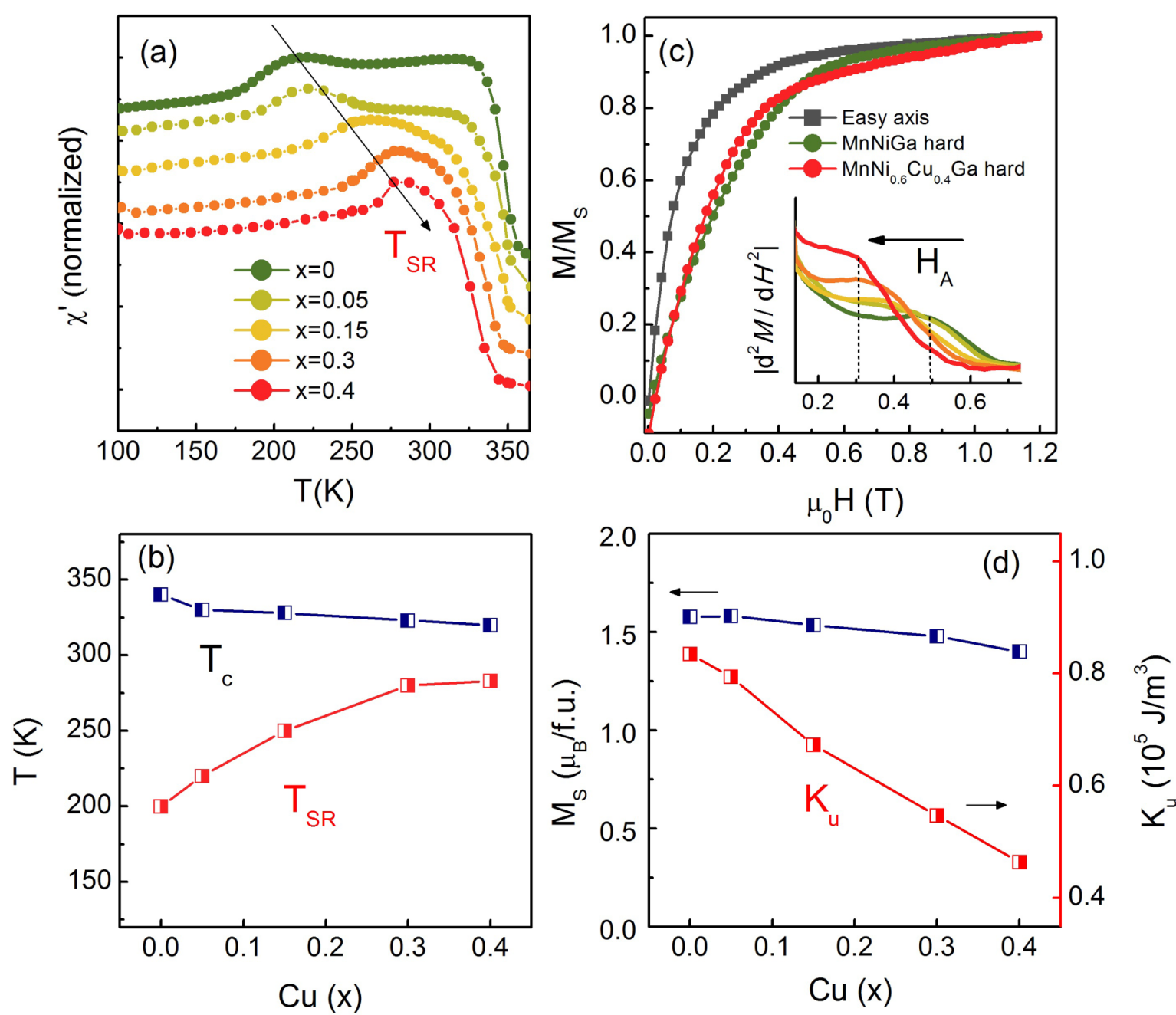

FIG. 2. (a) The real part of the ac susceptibility vs temperature and (b) the extracted Curie temperature $T_{\mathrm{C}}$ and spin reorientation temperature $T_{\mathrm{SR}}$ of the same compositions. (c) RT $M-H$ curve along the easy and hard axis of the magnetically aligned samples of $x=0$ and 0.4 . Inset in (c) is the $d^{2} M / d H^{2}$ vs $H$ curve for magnetization in the hard axis, and the abscissa of the peaks are determined as $H_{\mathrm{A}}$ according to the principle of SPD. (d) RT saturated magnetic moment and the derived uniaxial anisotropy constant for the above compositions.

in a cylinder tube at a magnetic field of $1 \mathrm{~T}$. The $\mathrm{x}$-ray diffraction (XRD) results of the original and aligned samples were compared to determine the crystal direction of the easy axis. By applying the magnetic field perpendicular and parallel to the aligned sample, the anisotropy field was obtained using the singular point detection (SPD) method $[32,33]$.

The thin plates for Lorentz TEM observations were cut from bulk polycrystalline samples and thinned by mechanical polishing and argon-ion milling. The Lorentz TEM measurements were performed in Tecnai F20 by using the Lorentz TEM mode and a JEOL-dedicated Lorentz TEM, both equipped with liquid-nitrogen, low-temperature holders $(\sim 100 \mathrm{~K})$ to study the temperature dependence of the magnetic textures. A magnetic field was applied perpendicular to the thin samples, and its magnitude was controlled by tuning the electric current of the objective lens.

Micromagnetic simulations were carried out by using a three-dimensional object oriented micromagnetic framework (OOMMF) code based on the Landau-Lifshitz-Gilbert (LLG) function [34]. The energy terms include only the uniaxial anisotropy, exchange, demagnetization, and Zeeman energy.
A model of $400 \times 400 \times 200 \mathrm{~nm}^{3}$ was adopted for the single skyrmion, with a mesh size of $5 \mathrm{~nm}$. A damping constant of $\alpha=1$ was applied to ensure a quick relaxation to the equilibrium state.

\section{RESULTS AND DISCUSSION}

The synchrotron radiation $\mathrm{x}$-ray diffractions showed that the arc-melted $\mathrm{MnNi}_{1-x} \mathrm{Cu}_{x} \mathrm{Ga} \quad(x=0-1.0)$ alloys exhibit a pure $\mathrm{Ni}_{2} \mathrm{In}$ structure when the $\mathrm{Cu}$-substitution content $x$ is below 0.5 [Figs. 1(b) and 1(c)]. As the x-ray scattering lengths of $\mathrm{Mn}, \mathrm{Ni}$, and $\mathrm{Cu}$ are close to each other, the atomic occupations are mainly examined by the later-discussed neutron diffractions, which showed that the $\mathrm{Cu}$ atoms prefer to occupy the Ni site [Fig. 1(a)]. The overall lattice constants increase with $\mathrm{Cu}$ substitution, while decreasing with lowering temperature, as seen in Figs. 1(c) and 1(d). Remarkably, we notice that while $a$ and $c$ grow uniformly with the increasing temperature in this range (see Fig. S1 in the Supplemental Material (SM) [35]), the $c / a$ vs $T$ curves show kinks at certain temperatures, indicted by the hollow squares in Fig. 1(d). As 


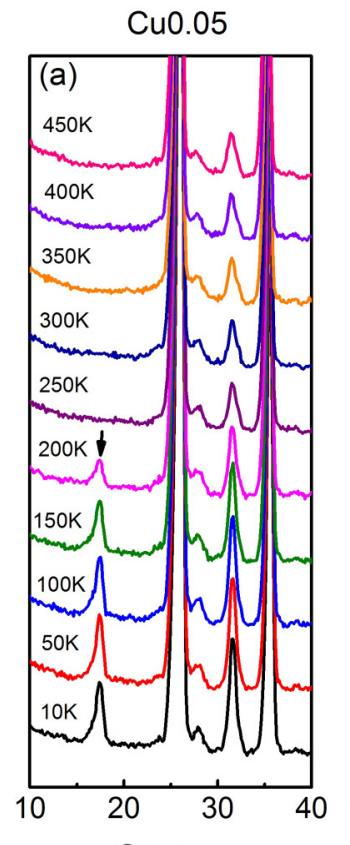

$2 \theta\left({ }^{\circ}\right)$
Cu0.3

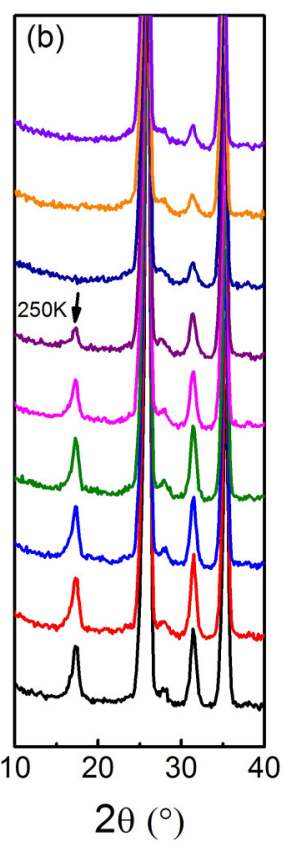

Cu0.15

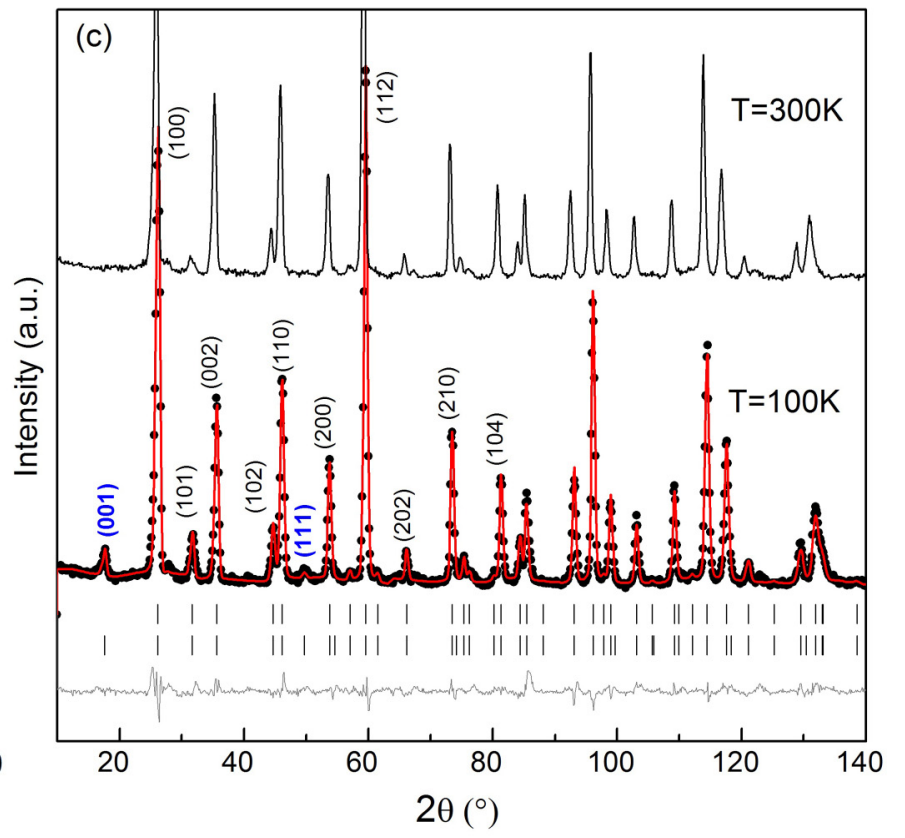

FIG. 3. (a), (b) Temperature-dependent evolutions of the (001) peak for $x=0.05$ (a) and 0.3 (b). (c) Observed (black) and fitted neutron diffraction patterns (red) and their difference profiles (gray) for $\mathrm{Cu}(x=0.15)$ at 300 and $100 \mathrm{~K}$. Vertical lines indicate the peak positions for the nuclear (top) and magnetic (bottom) reflection of the hexagonal MnNiGa phase. The emergence of (001) and (111) diffractions is related to the AFM component in the basal plane.

the variable-temperature XRD measurements have excluded the occurrence of structural transition with lowering temperature for these alloys, the kink should be associated with certain magnetic structure changes.

The magnetic structures are first investigated by the macroscopic magnetization measurements. The temperature dependences of ac susceptibility for $x=0,0.05,0.15,0.3$, and 0.4 alloys are presented in Fig. 2(a). The bump is regarded as an indication of a spin reorientation transition, which has already been reported in $\mathrm{MnNiGa}$ [12]. The changes of SRT temperature $T_{\mathrm{SR}}$ and Curie temperature $T_{\mathrm{C}}$ are shown in Fig. 2(b). It is noted that the $T_{\mathrm{SR}}$ systematically shifts upwards with $\mathrm{Cu}$ substitution, while $T_{\mathrm{C}}$ is lowered a little and maintained at higher than room temperature (RT). The $T_{\mathrm{SR}}$ also accords to the kink temperature of $c / a$ ratio, confirming the magnetic origin of the structural distortion. The variabletemperature $M-H$ curves were also measured for these alloys, which showed similar magnetization behavior. The saturated magnetization presents no substantial decrease upon the nonmagnetic $\mathrm{Cu}$ substitution (SM Fig. S2 [35]). Particularly, the magnetocrystalline anisotropy properties were examined by magnetic alignment of the polycrystalline samples (for details see the Methods section). It is revealed that the easy axes of all the alloys are along the (002) direction, i.e., $c$ axis. Then the magnetization curves in the hard and easy axis are measured, with the anisotropy field $H_{\mathrm{A}}$ determined by the SPD method [32,33], as seen in Fig. 2(c). The derived uniaxial anisotropy constant $K_{u}=\frac{1}{2} \mu_{0} H_{\mathrm{A}} M_{\mathrm{S}}$, as exhibited in Fig. 2(d), is found to decrease monotonically with the $\mathrm{Cu}$ substitution.

To figure out the exact magnetic structure accompanied with the occurrence of SRT, as well as the possible relation between the magnetocrystalline anisotropy and the internal magnetic structure, the powder neutron diffractions on the samples of $x=0,0.05,0.15$, and 0.3 were performed from 400 (above $T_{\mathrm{C}}$ ) to $10 \mathrm{~K}$. At the temperatures above $T_{\mathrm{C}}$, where only the nuclear scattering contributes to the neutron diffraction pattern, the precise crystal structure and atomic occupation can be determined. As seen in the SM Fig. S3 [35] and Fig. 3, The main peaks are indexed to the $\mathrm{Ni}_{2} \mathrm{In}$ structure (space group P63/mmc, 194), consistent with the above XRD data. The atom arrangement corresponding to the minimum disagreement factors is as follows: the $2 a$ site is entirely occupied by the Mn atoms, the $2 c$ site by the Ga atoms, the $2 d$ site is together occupied by the original $\mathrm{Ni}$ atoms and doping $\mathrm{Cu}$ atoms, which partly accords to the previous studies on $\left(\mathrm{Mn}_{1-x} \mathrm{Ni}_{x}\right)_{65} \mathrm{Ga}_{35}$ [36] and $\left(\mathrm{Mn}_{1-x} \mathrm{Cu}_{x}\right)_{66} \mathrm{Ga}_{34}$ [37] alloys. Due to the close valence electrons of $\mathrm{Cu}$ and $\mathrm{Ni}$, they are nearly mixed uniformly at the $2 d$ site.

Besides the nuclear contribution, the magnetic structure can be acquired by taking into account the magnetic contribution at temperatures below $T_{C}$. Notably, extra peaks of (001) and (111) appeared when the temperature is lowered to $200 \mathrm{~K}$ for $x=0,0.05$ and 0.15 , and to $250 \mathrm{~K}$ for $x=0.3$, as seen in Fig. 3. In addition, the transition temperature and its variations with composition coincide to the above observed $T_{\mathrm{SR}}$ in the ac susceptibility curve, confirming the occurrence of the spin reorientation transition characterized by the bump. Above $T_{\mathrm{SR}}$, the diffraction positions are fully superimposed with the pure nuclear contribution; thus a magnetic cell with a collinear ferromagnetic (CFM) structure is proposed and the refined moments are all oriented to the $c$ axis. This is consistent with the $c$-axis anisotropy by the MCA measurement. The 
(a)
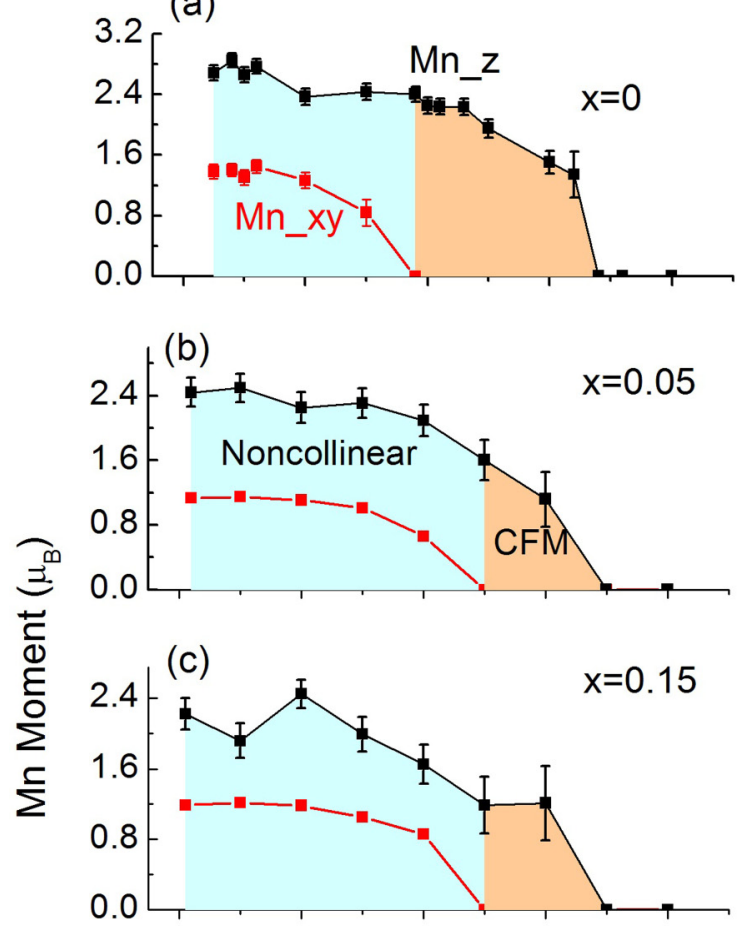

(d)

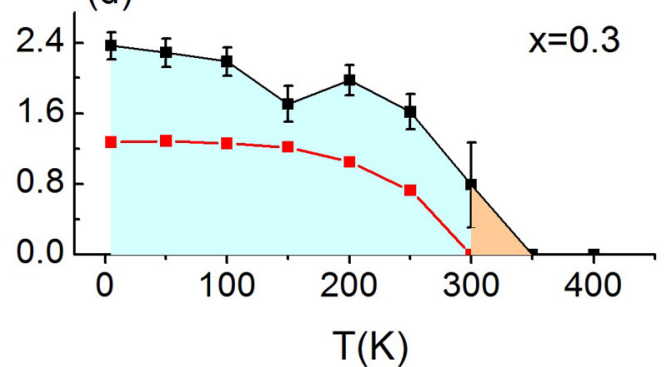

(e)
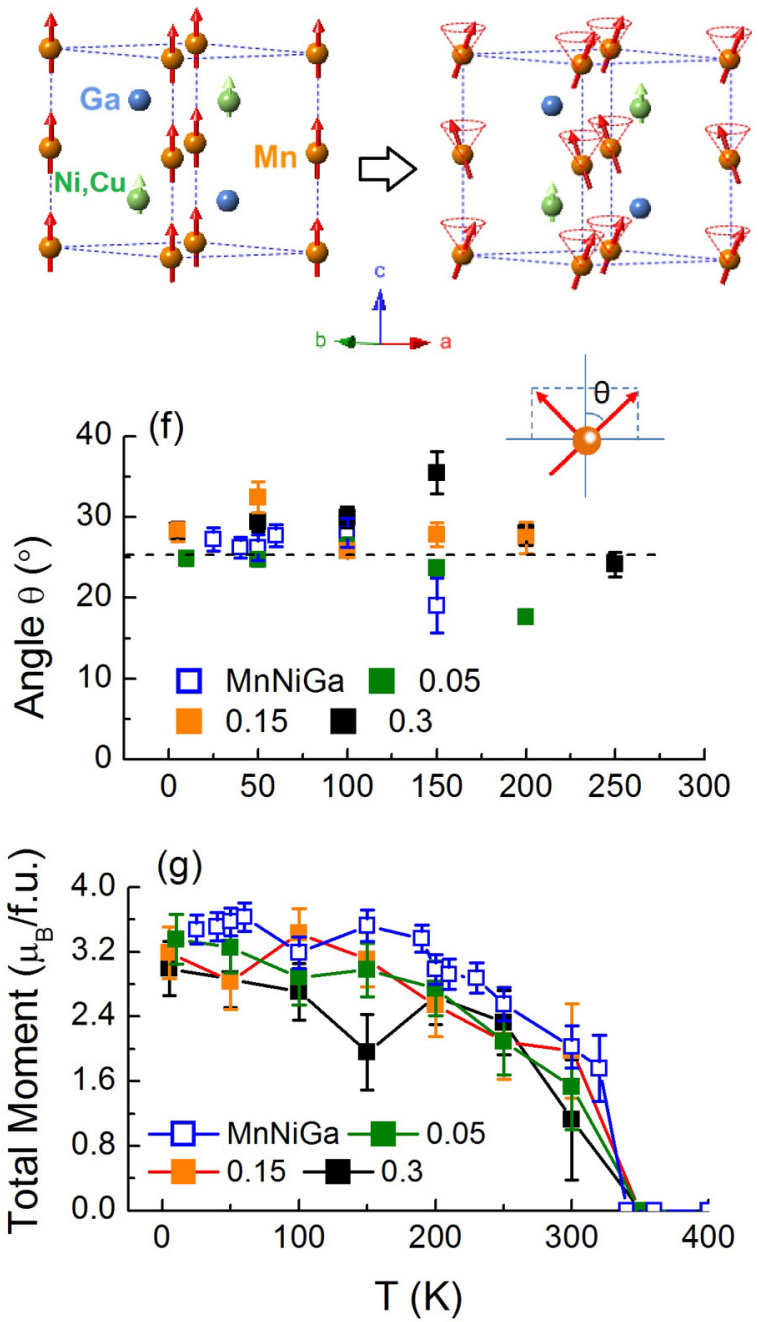

FIG. 4. (a)-(d) Temperature dependence of the refined magnetic moments of the Mn atom for $x=0,0.05,0.15$, and 0.3 alloys. (e) Schematic of the refined magnetic structure at high and low temperatures. (f), (g) Temperature dependence of the tilt angle $\theta$ and the total magnetic moment, respectively.

emergence of a (001) peak at lower temperature signifies the appearance of an antiferromagnetic (AFM) component lying in the basal plane. Since the peak position still belongs to the magnetic reflection at a propagation vector of $k=$ $[0,0,0]$ based on the symmetry analyses, the AFM coupling can take place between the atoms inside one crystal cell (possible magnetic configurations are listed in SM Table S1 [35]). In combination with the magnetization value obtained in the variable-temperature $M-H$ curves, we proposed the noncollinear magnetic structure shown in Fig. 4(e), with the Mn moment canted along $c(z)$ axis and the Ni moment parallel to the $c$ component of the Mn atom.

As the Mn atoms carry the main moment and constitute the canting structure, their changes are summarized in Figs. 4(a)4(d). The Ni moments range from 0.6 to $1 \mu_{\mathrm{B}}$ (SM Fig. S4 [35]). In the noncollinear magnetic structure, the moments along the $c$ axis and $a b$ plane are denoted as $\mathrm{Mn}_{z}$ and $\mathrm{Mn}_{x y}$ ( $a$ and $b$ axes are taken equivalent and not resolved), respectively. It can be seen that $\mathrm{Mn}_{z}$ lies around $2.4 \mu_{\mathrm{B}}$ and $\mathrm{Mn}_{x y}$ is about $1.2 \mu_{\mathrm{B}}$ at $10 \mathrm{~K}$, and it results in a canting angle $\theta$ of $\sim 25^{\circ}$ [Fig. 4(f)]. Unexpectedly, the canting degree varies little with respect to temperature, which means that the canting takes place abruptly rather than in a gradual process. The overall magnetic structure varies little upon $\mathrm{Cu}$ substitution, as seen in both the magnitude of the total magnetization [Fig. 4(g)] and the atomic resolved value, except that the spin-canting region is broadened, in agreement with the trend of SRT. Based on the refined magnetic structure, enhance of $T_{\mathrm{SR}}$ means the advance of the spin-direction deviation from the $c$ axis, therefore meaning the lowering of $c$-axis anisotropy with $\mathrm{Cu}$ substitution, consistent with the above macroscopy measurements. The reduction of MCA can be attributed to the weakened Mn-Ni-Mn FM coupling by the substitution. Additionally, the emergence of AFM coupling indicates the existence of FM and AFM competition between the two Mn sublattices here $[38,39]$, which can facilitate the formation of biskyrmions according to theoretical prediction [16].

To check how magnetic structure variation affects the formation of biskyrmions, Lorentz TEM observations were performed for the above alloys. It is found that in the samples 


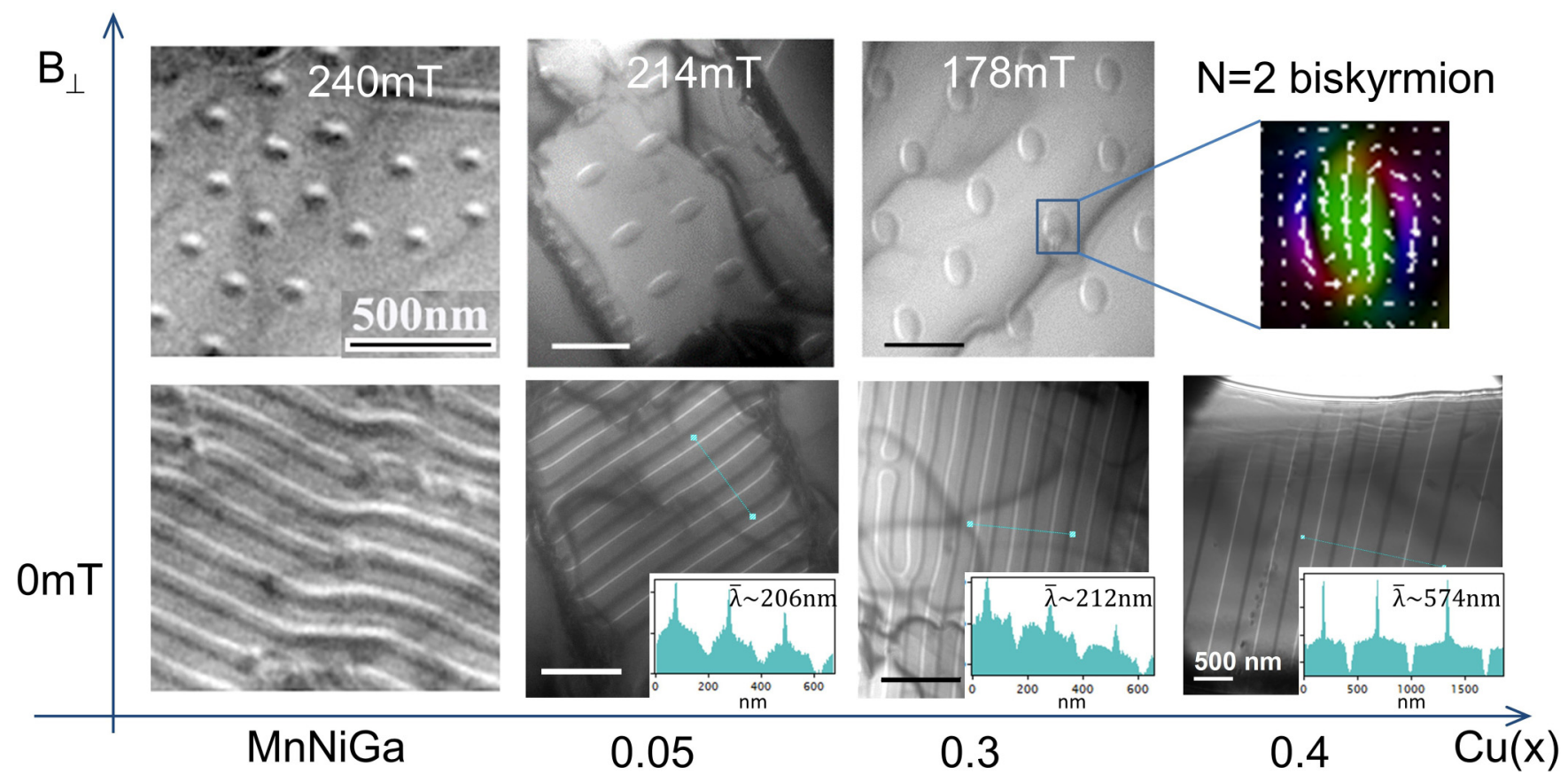

FIG. 5. Lorentz TEM images for MnNiGa (adapted from Ref. [12]) and doping content of $x=0.05,0.3$, and 0.4 at RT, at zero field (lower panel), and external fields (upper panel indicated in the figure). Magnified picture is the transport-of-intensity equation of the spin texture of the magnetic domain. Insets of the stripe domains show the Lorentz contrast along the path marked by the dotted green line.

of $x=0-0.3$, similar dense stripe domains develop at zero field and biskyrmions can form upon increasing magnetic field at RT, as shown in Fig. 5 for $x=0.05$ and 0.3. The biskyrmion reveal the typical double-helicity spin texture, which is confirmed by the transport-of-intensity (TIE) analyses and is characterized by the elliptical morphologies. Statistically, the stripe period is around $200 \mathrm{~nm}$ for all samples in this set (inset of Fig. 5), similar to that of MnNiGa [12]. Neither the stripe domain nor the biskyrmion texture was observed to present significant change among the alloys of $x=0-0.3$. This might be because the domain dimension roughly scales with the characteristic materials length $l=\frac{\gamma_{\mathrm{w}}}{\mu_{0} M_{\mathrm{S}}^{2}}$, where $\gamma_{w}$ is the domain wall energy and is proportional to $\sqrt{A K_{\mathrm{u}}}$ [40]; thus a small change of $K_{\mathrm{u}}$ or $A$ can actually generate no obvious change in the domain structures, as also proved by our previous simulations [13]. However, in $x=0.4$, a distinct large-width stripe $(\sim 570 \mathrm{~nm})$ is identified at zero field, and the skyrmion formation is absent upon increasing field. The line scan of the stripe domains showed that the domain walls of $x=0.05$ and 0.3 are thicker and the magnetization in the interval varies continually (especially in $x=0.05$ ), representing the typical ground states that tend to form bubblelike domains [26,41], while at $x=0.4$, the Lorentz contrast is sharp across the wall and a large quantity of spins lie stably in the film plane. The wide in-plane stripes are not favorable to accommodate skyrmion or bubble domains under the field but only become narrowed until disappearing, like those observed in Ref. [41]. This dramatic change signifies the $K_{\mathrm{u}}$ at $x=0.4$ might reach a critical point of being out-of-plane or in-plane.

We also inspect the influence of SRT on the skyrmion statics by varying-temperature Lorentz TEM measurements.
As seen in SM Fig. S5 [35], the biskyrmions can explicitly nucleate both below and above SRT temperature in $x=0.3$, which takes place in the other three samples as well. The critical field of stripe-skyrmion transformation smoothly lowers with increasing temperature, which is well explained by the thermal-fluctuation-assisted skyrmion stabilization [4,5]. No anomaly was resolved presently to be associated with the SRT. Neither the stripe period (here about $300 \mathrm{~nm}$, in another set of samples) nor the skyrmion morphology seems to be affected by the SRT. This means that the mesoscopic domains exhibit no direct dependence on a specific noncollinear magnetic structure at an atomic scale but more explicitly rely on the change of macro properties, like the MCA. The importance of the SRT resides in that on one side it reflects the existence of FM and AFM competition, while on the other side, it helps explain the origin of reducing MCA by the substitution of $\mathrm{Cu}$ atoms.

Based on the above results, a proper MCA is necessary to accommodate biskyrmions, as also illustrated in several skyrmion systems $[13,42]$. But the criterion for the formation of a biskyrmion can be more subtle. For example, a small substitution of $\mathrm{Ga}$ by $\mathrm{Y}$ can turn MnNiGa into a trivial bubble [20]. Here we performed micromagnetic simulations to establish a parameter space for biskyrmions. The saturation magnetization $\mathrm{M}_{\mathrm{S}}$ is chosen as the ground value of $\mathrm{MnNiGa}$, with $6.1 \times 10^{5} \mathrm{~A} / \mathrm{m}$. The exchange constant is varied from 1.0 to $2.0 \times 10^{-11} \mathrm{~J} / \mathrm{m}$. A magnetic field of $200 \mathrm{mT}$ is applied to the model to stabilize the skyrmion state. The initial configuration of the domain is set as a prototype biskyrmion with $N=2$. We find that after relaxation, it turns to a trivial bubble with $N=0$ under relatively large uniaxial anisotropy values $\left(K_{\mathrm{u}} \sim 0.5 \times 10^{5} \mathrm{~J} / \mathrm{m}^{3}\right.$, when $\left.A=1.0 \times 10^{-11} \mathrm{~J} / \mathrm{m}\right)$, 


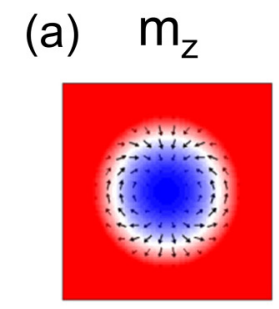

(c)
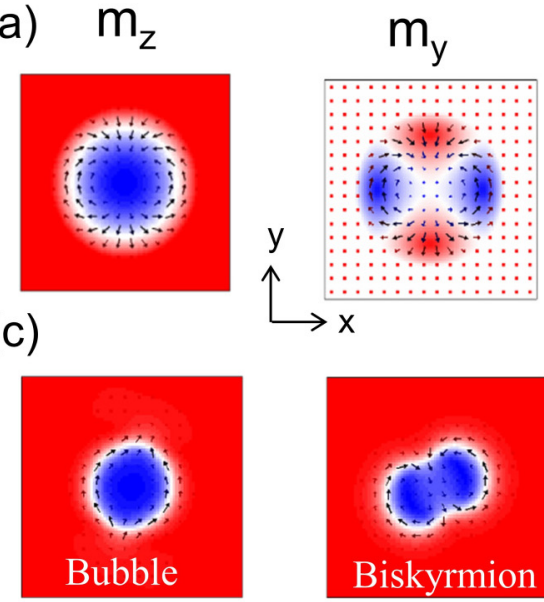

(d)
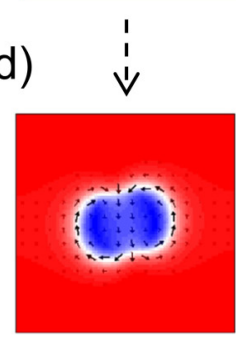
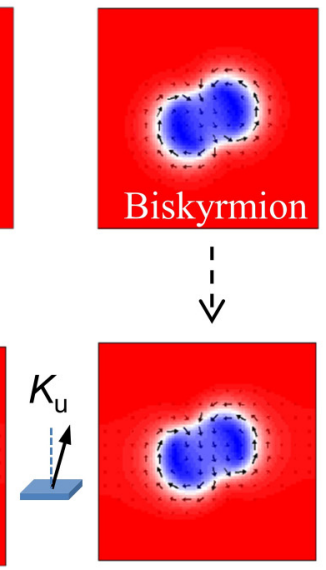

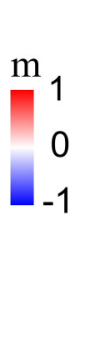

0.5

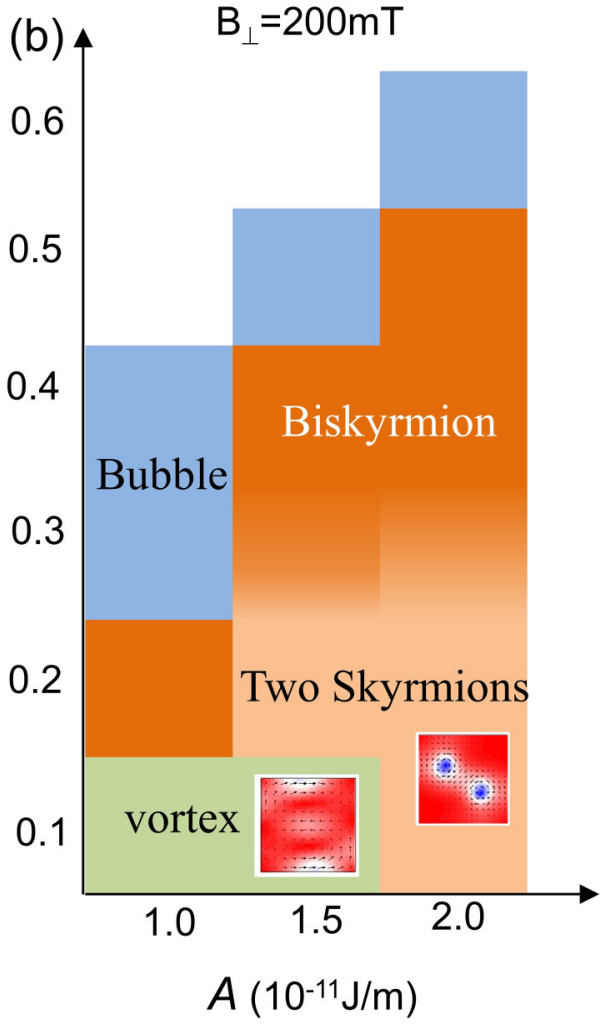

FIG. 6. Micromagnetic simulation of the stability of a single biskyrmion. Model size is $400 \times 400 \times 200 \mathrm{~nm}^{3}$. The external field is $200 \mathrm{mT}$ along the $z$ axis. (a) The initial state of an ideal biskyrmion structure. (b) Variation of the domain structures by changing $A$ and $K_{\mathrm{u}}$. (c), (d) A trivial bubble and biskyrmion after relaxation and their evolution under a tilt of $K_{\mathrm{u}}\left(10^{\circ}\right)$ from the perpendicular direction.

as shown in Fig. 6. Notably, the biskyrmion state can be recovered when the $K_{\mathrm{u}}\left(=0.6 \times 10^{5} \mathrm{~J} / \mathrm{m}^{3}\right)$ is tilted away from the perpendicular direction [Fig. 6(d)]. This phenomenon has been observed in the study of the crystal direction dependence of the domains in off-stoichiometric $\left(\mathrm{Mn}_{1-x} \mathrm{Ni}_{x}\right)_{65} \mathrm{Ga}_{35}$ alloys [26]. With lowering $K_{\mathrm{u}}$, the biskyrmion state can be stabilized under a certain field and maintained when $K_{\mathrm{u}}$ is tilted. But too small $K_{\mathrm{u}}$ leads to an in-plane stripe that will evolve into a vortex under field. Therefore, an elaborate adjustment of $K_{\mathrm{u}}$ is proved to be critical for producing biskyrmions. Here it should be pointed out that the exact critical values of $K_{\mathrm{u}}$ given by the simulations might deviate from that of experiments, for example, in $x=0.4\left(K_{\mathrm{u}} \sim 0.46 \times 10^{5} \mathrm{~J} / \mathrm{m}^{3}\right)$, which is a limitation caused by the idealization and simplification of the theoretical model. But the general trends of the influence of MCA on the biskyrmion formation are consistent with each other. Also, the FM-AFM competition revealed in the magnetic structure is another important factor for the formation of high-topological-charge skyrmions [16]. These two conditions are simultaneously fulfilled in our system, thus making it a good biskyrmion host.

\section{CONCLUSIONS}

We have presented a detailed study of the magnetic structure of single-phased $\mathrm{MnNi}_{1-x} \mathrm{Cu}_{x} \mathrm{Ga} \quad(x=0-0.4)$ alloys by the magnetization measurements and neutron diffractions, where a noncollinear canted ferromagnetic structure was uncovered below a spin reorientation transition temperature. Nonmagnetic $\mathrm{Cu}$ substitution at the Ni site can continuously tune $T_{\mathrm{SR}}$ while maintaining the high $T_{\mathrm{C}}$ above RT. It is found that spin reorientation is greatly associated with the change of MCA and signifies the existence of AFM-FM competition in this system. The biskyrmion formation is observed by Lorentz TEM in the compositions of $x=0-0.3$, where the uniaxial anisotropy is anticipated to be located in a proper range. Our work has further helped clarify the origin of biskyrmion formation in materials and prospectively offered a guideline to fine-tune the skyrmion topology.

\section{ACKNOWLEDGMENTS}

This work was supported by the National Natural Science Foundation of China (Grant No. 11604148), the National Key R\&D Program of China (Grant No. 2017YFA0303202), and the Key Research Program of the Chinese Academy of Sciences, KJZD-SW-M01. Z.X.C. thanks the Australia Research Council for support (DP190100150). We also would like to acknowledge Dr. Junye Yang at the Paul Scherrer Institute, Switzerland, and Dr. Qiang Zhang at King Abdullah University of Science and Technology (KAUST), Saudi Arabia, for technical support and helpful discussions. 
[1] U. K. Rößler, A. N. Bogdanov, and C. Pfleiderer, Nature (London) 442, 797 (2006).

[2] X. Z. Yu, N. Kanazawa, W. Z. Zhang, T. Nagai, T. Hara, K. Kimoto, Y. Matsui, Y. Onose, and Y. Tokura, Nat. Commun. 3, 988 (2012).

[3] A. Fert, V. Cros, and J. Sampaio, Nat. Nanotechnol. 8, 152 (2013).

[4] S. Mühlbauer, B. Binz, F. Jonietz, C. Pfleiderer, A. Rosch, A. Neubauer, R. Georgii, and P. Böni, Science 323, 915 (2009).

[5] X. Z. Yu, N. Kanazawa, Y. Onose, K. Kimoto, W. Z. Zhang, S. Ishiwata, Y. Matsui, and Y. Tokura, Nat. Mater. 10, 106 (2010).

[6] W. Münzer, A. Neubauer, T. Adams, S. Mühlbauer, C. Franz, F. Jonietz, R. Georgii, P. Böni, B. Pedersen, M. Schmidt, A. Rosch, and C. Pfleiderer, Phys. Rev. B 81, 041203(R) (2010).

[7] O. Boulle, J. Vogel, H. Yang, S. Pizzini, D. de Souza Chaves, A. Locatelli, T. O. Menteş, A. Sala, L. D. Buda-Prejbeanu, and O. Klein, Nat. Nanotechnol. 11, 449 (2016).

[8] W. J. Jiang, G. Chen, K. Liu, J. D. Zang, S. G. E. te Velthuis, and A. Hoffmann, Phys. Rep. 704, 1 (2017).

[9] S. Heinze, K. von Bergmann, M. Menzel, J. Brede, A. Kubetzka, R. Wiesendanger, G. Bihlmayer, and S. Blugel, Nat. Phys. 7, 713 (2011).

[10] X. Yu, M. Mostovoy, Y. Tokunaga, W. Zhang, K. Kimoto, Y. Matsui, Y. Kaneko, N. Nagaosa, and Y. Tokura, Proc. Natl. Acad. Sci. USA 109, 8856 (2012).

[11] X. Z. Yu, Y. Tokunaga, Y. Kaneko, W. Z. Zhang, K. Kimoto, Y. Matsui, Y. Taguchi, and Y. Tokura, Nat. Commun. 5, 3198 (2014)

[12] W. Wang, Y. Zhang, G. Xu, L. Peng, B. Ding, Y. Wang, Z. Hou, X. Zhang, X. Li, E. Liu, S. Wang, J. Cai, F. Wang, J. Li, F. Hu, G. Wu, B. Shen, and X. X. Zhang, Adv. Mater. 28, 6887 (2016).

[13] Z. Hou, W. Ren, B. Ding, G. Xu, Y. Wang, B. Yang, Q. Zhang, Y. Zhang, E. Liu, F. Xu, W. Wang, G. Wu, X. Zhang, B. Shen, and Z. Zhang, Adv. Mater. 29, 1701144 (2017).

[14] X. Yu, Y. Tokunaga, Y. Taguchi, and Y. Tokura, Adv. Mater. 29, 1603958 (2017).

[15] N. Nagaosa and Y. Tokura, Nat. Nanotechnol. 8, 899 (2013).

[16] X. Zhang, J. Xia, Y. Zhou, X. Liu, H. Zhang, and M. Ezawa, Nat. Commun. 8, 1717 (2017).

[17] L. Peng, Y. Zhang, W. Wang, M. He, L. Li, B. Ding, J. Li, Y. Sun, X. G. Zhang, J. Cai, S. Wang, G. Wu, and B. Shen, Nano Lett. 17, 7075 (2017).

[18] B. Ding, Y. Li, G. Xu, Y. Wang, Z. Hou, E. Liu, Z. Liu, G. Wu, and W. Wang, Appl. Phys. Lett. 110, 092404 (2017).

[19] L. Peng, Y. Zhang, M. He, B. Ding, W. Wang, J. Li, J. Cai, S. Wang, G. Wu, and B. Shen, J. Phys.: Condens. Matter 30, 065803 (2018).

[20] S. L. Zuo, Y. Zhang, L. C. Peng, X. Zhao, R. Li, H. Li, J. F. Xiong, M. He, T. Y. Zhao, J. R. Sun, F. X. Hu, and B. G. Shen, Nanoscale 10, 2260 (2018).

[21] R. Takagi, X. Z. Yu, J. S. White, K. Shibata, Y. Kaneko, G. Tatara, H. M. Ronnow, Y. Tokura, and S. Seki, Phys. Rev. Lett. 120, 037203 (2018).
[22] X. F. Xiao, L. C. Peng, X. G. Zhao, Y. Zhang, Y. Y. Dai, J. Guo, M. Tong, J. Li, B. Li, W. Liu, J. W. Cai, B. G. Shen, and Z. D. Zhang, Appl. Phys. Lett. 114, 142404 (2019).

[23] X. Li, S. Zhang, H. Li, D. A. Venero, J. S. White, R. Cubitt, Q. Huang, J. Chen, L. He, G. van der Laan, W. Wang, T. Hesjedal, and F. Wang, Adv. Mater. 31, 1900264 (2019).

[24] Y. Yao, B. Ding, J. Cui, X. Shen, Y. G. Wang, W. H. Wang, and R. C. Yu, Appl. Phys. Lett. 114, 102404 (2019).

[25] J. C. Loudon, A. C. Twitchett-Harrison, D. Cortes-Ortuno, M. T. Birch, L. A. Turnbull, A. Stefancic, F. Y. Ogrin, E. O. Burgos-Parra, N. Bukin, A. Laurenson, H. Popescu, M. Beg, O. Hovorka, H. Fangohr, P. A. Midgley, G. Balakrishnan, and P. D. Hatton, Adv. Mater. 31, 1806598 (2019).

[26] B. Ding, H. Li, X. Li, Y. Wang, Z. Hou, G. Xu, E. Liu, G. Wu, F. Wang, and W. Wang, APL Mater. 6, 076101 (2018).

[27] Y. Tokunaga, Y. Kaneko, D. Okuyama, S. Ishiwata, T. Arima, S. Wakimoto, K. Kakurai, Y. Taguchi, and Y. Tokura, Phys. Rev. Lett. 105, 257201 (2010).

[28] T. Fukumura and H. Sugawara, Science 284, 1969 (1999).

[29] A. K. Nayak, V. Kumar, T. Ma, P. Werner, E. Pippel, R. Sahoo, F. Damay, U. K. Rossler, C. Felser, and S. S. P. Parkin, Nature (London) 548, 561 (2017).

[30] H. Rietveld, J. Appl. Crystallogr. 2, 65 (1969).

[31] J. Rodriguez Carvajal, Physica B 192, 55 (1993).

[32] G. Asti and S. Rinaldi, Phys. Rev. Lett. 28, 1584 (1972).

[33] R. Scholl, K. Elk, and L. Jahn, J. Magn. Magn. Mater. 82, 235 (1989).

[34] M. J. Donahue and D. G. Porter, OOMMF User's Guide, version 1.0; Interagency Report NISTIR 6376, National Institute of Standards and Technology, 1999.

[35] See Supplemental Material at http://link.aps.org/supplemental/ 10.1103/PhysRevB.100.054416 for additional data for the structural parameters, magnetization, LTEM observation, and detailed neutron diffraction analyses.

[36] H. Shiraishi, H. Niida, Y. Iguchi, S. Mitsudo, M. Motokawa, K. Ohayama, H. Miki, H. Onodera, T. Hori, and K. Kanematsu, J. Magn. Magn. Mater. 196-197, 660 (1999).

[37] H. Shiraishi, Y. Iguchi, K. Ohoyama, Y. Yamaguchi, K. Shimizu, and T. Hori, J. Magn. Magn. Mater. 226-230, 1081 (2001).

[38] Y. R. You, G. Z. Xu, J. X. Tang, Y. Y. Gong, and F. Xu, Intermetallics 106, 88 (2019).

[39] O. Meshcheriakova, S. Chadov, A. K. Nayak, U. K. Rößler, J. Kübler, G. André, A. A. Tsirlin, J. Kiss, S. Hausdorf, A. Kalache, W. Schnelle, M. Nicklas, and C. Felser, Phys. Rev. Lett. 113, 087203 (2014).

[40] A. Hubert and R. Schäfer, Magnetic Domains (Springer-Verlag, Berlin, 1998), pp. 175-177.

[41] A. Kotani, H. Nakajima, K. Harada, Y. Ishii, and S. Mori, Phys. Rev. B 94, 024407 (2016).

[42] S. A. Montoya, S. Couture, J. J. Chess, J. C. T. Lee, N. Kent, D. Henze, S. K. Sinha, M. Y. Im, S. D. Kevan, P. Fischer, B. J. McMorran, V. Lomakin, S. Roy, and E. E. Fullerton, Phys. Rev. B 95, 024415 (2017) 